Submission ID: 43902

\title{
Reflected and Scattered Components Extraction from Migrated Wave Field
}

A.A. Shevchenko* (PetroTrace), B.S. Esinov (PetroTrace), O.O. Litvyakova (PetroTrace), A.E. Korolev (PetroTrace), K.A. Smirnov (PetroTrace), E. Landa (Tel Aviv University)

\section{SUMMARY}

The diffraction component of the wave field gives significant information about the position of faults and fractured areas. There are different approaches for wave field separation into reflected and scattered components. For directional gathers obtained by full-azimuth migration there are also different ways of data filtering. The methods that are used for production projects as well as new developments are discussed in this report. 


\section{Разделение мигрированных волновых полей на отраженную и рассеянную компоненты}

Б.С. Есинов (ООО «ПетроТрейс»), О.О. Литвякова (ООО «ПетроТрейс»), А.Е. Королев (ООО «ПетроТрейс»), К.А. Смирнов (ООО «ПетроТрейс»), А.А. Шевченко* (ООО «ПетроТрейс») Е. Ланда (Тель-Авививский университет)

На стадии постмиграционной обработки и интерпретации существенную информацию о положении разломов, зон трещиноватости дает рассеянная или дифракционная компонента волнового поля. Существуют различные подходы к разделению поля на отраженную и рассеянную составляющие. При обработке дирекционных сейсмограмм, получаемых после применения полноазимутальной угловой миграции, существуют различные пути фильтрации данных. В докладе обсуждаются программы и методики, применяемые в производственных проектах и новые разработки. Приводятся примеры удачных и неудачных попыток разделения полей.

\section{Введение}

Исследования, посвященные выделению неоднородностей геологического разреза по сейсмическим волновым, имеют достаточно длинную историю. Последние публикации отражают часть подходов и методик изучения обсуждаемой темы Ribet et al. (2017), Hoeber et al. (2017), Koltanovsky et al. (2017), Merzlikin et al. (2017), Pelissier et al. (2017), Zelewski et al. (2017). Так же, как и для построения изображений по отраженным волнам (миграции) существует много объективно хороших, но алгоритмически различных программ, так и при построении дифракционных изображений алгоритмы построения могут различаться, но давать похожий результат. Выделение волны из полного волнового поля, обычно выполняемое методами пространственной фильтрации, основывается на предположении об известной форме годографа выделяемой волны. Если точно не известна форма годографа, или в волновом поле присутствуют несколько волн, то алгоритмы фильтрации должны основываться на знании некоторых симметрий годографов выделяемых волн Harlan et al. (1984), Blyas (2007). Мы развиваем подход к разделению отраженного и дифрагированного волновых полей, предложенный в работе Landa et al. (2008). В применении к трехмерным данным подход позволяет выполнять выделение дифрагированной компоненты сейсмической записи в зависимости от выбранного азимута сейсмограммы общей точки изображения (CIG).

\section{Метод}

Для разделения волновых полей на отражённую и дифрагированную компоненты в случае 3Д сейсмограмм используется методика, описанная в статье Landa et al. (2008). Приведенные в статье формулы годографов отраженной и дифрагированной волн для случая 2Д, применяются для азимутальных сечений $3 Д$ сейсмограммы CIG. При таком подходе преобразования Радона по двухмерным сечениям поля работают в предположении о наличии рассеивающего объекта в выбранном азимуте. На Рис.1А приведен пример азимутального сечения 3Д сейсмограммы после миграции. На двумерном сечении годографы отраженных волн имеют максимум в окрестности центра сейсмограммы и время годографа убывает с увеличением угла наклона нормали к отражающей площалке. По теории годографы дифрагированных волн имеют форму близкую к горизонтальной. На Рис.1Б и В приведен пример разделения волнового поля на две компоненты (отражение -Б и дифракция В). Алгоритм фильтрации поля основан на итеративном выделении из поля регулярных событий, имеющих заданную форму годографа. Как правило сходимость итеративных алгоритмов для выделения одной компоненты регулярного поля работает очень хорошо (Harlan et al. (1984)). При наличии в волновом поле двух и более волн для итеративных методов разделения можно задавать некоторые ограничения, улучшающие сходимость алгоритма. Например, в работе Blyas (2007) предлагается задавать последовательность вычитания отдельных волн из общего поля, основываясь на значении энергии каждой волны. Такой подход оправдан для случая 
скважинной сейсморазведки, но может давать некорректный результат в рассматриваемой задаче. В нашей работе мы возложили решение вопроса об определении энергии волновых компонент и последовательности разделения компонент на пользователя. В предлагаемом методе можно определять тестированием какую волну вычитать первой и с какой силой. Критерием качества, на котором основывается выбор параметров разделения является наличие регулярных отражений в отраженной компоненте поля и отсутствие отражений в дифракционной компоненте поля. Субъективный подход такого решения конечно оставляет высокой вероятность ошибки, но контролируемое решение позволяет избежать плохих результатов разделения.

\section{Практическое применение}

Практическое применение алгоритма разделения волновых полей выполнялось в рамках нескольких производственных проектов. Методика использования предполагает использование миграции ES-360 (Парадайм) и получения дирекционных сейсмограмм. Дирекционные сейсмограммы используются для получения двумерных сейсмограмм общей точки изображения (CIG) c фиксированным азимутом. Получение азимутально ориентированных сейсмограмм CIG позволяет изучать различие энергии дифрагированных волн по различным направлениям, однако помехоустойчивость суммарного (по всем азимутам) изображения свободно от помех и артефактов. На Рис.2. приведены три фрагмента мигрированных изображений, на которых видно преимущество обработки по предложенной методике. На левом фрагменте (STACK) результат суммирования полного поля по одному выбранному азимуту. Центральный фрагмент рисунка (DIF_STACK) и левый фрагмент (REF_STACK) - это дифракционная компонента и поле отраженных волн, выделенные по сейсмограммам и просуммированные для получения изображений. Хорошо видно, что на дифрагированной компоненте поля наблюдаются события с наклоном близким к вертикали. Такие события возможно представляют собой отражения от разломов и могут характеризовать степень трещиноватости геологического разреза. На изображении могут присутствовать и артефакты, которые связаны с вариацией амплитуд на исходных сейсмограммах перед миграцией. Артефакты миграции могут иметь существенную амплитуду из-за того, что в разделении на компоненты был использован только один азимут из мигрированных сейсмограмм. Подавление артефактов и более достоверное выделение дифракционных событий возможно путем суммирования выделенных компонент изображений с различными азимутами. 


\section{EAGE}

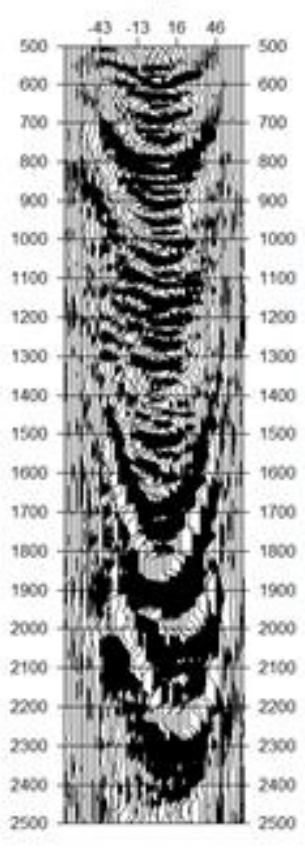

Б $\quad$ B

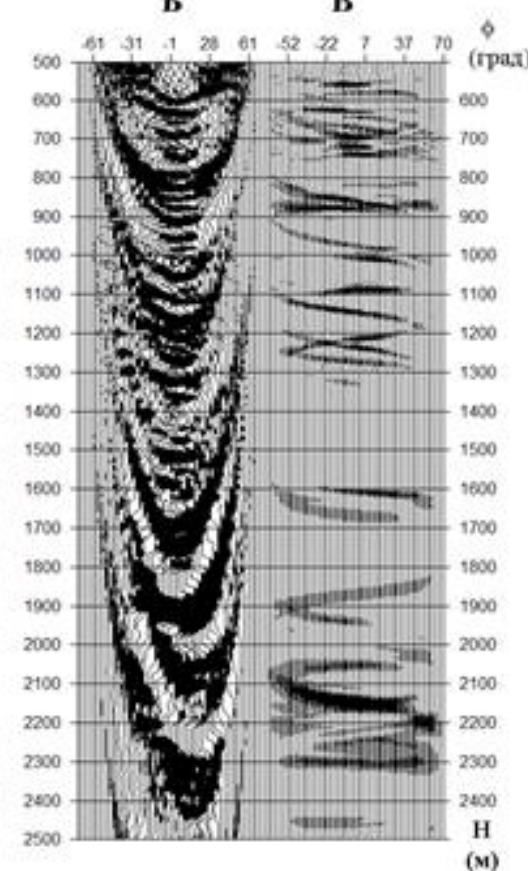

Рисунок 1. Пример обработки сейсмограммы общей точки изображения (CIG). A-исходная сейсмограмма 48 трасс с диапазоном углов -72 - +72. Б - Выделение модели отраженной компоненты. В - Выделение модели дифракиионной компоненты.
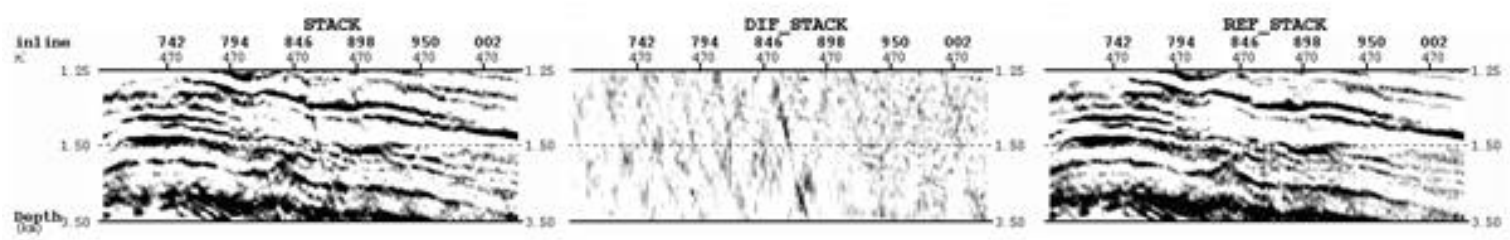

Рисунок 2. Пример фрагмента суммарного изображения после разделения на отраженную и дифракиионные компоненты.

\section{Выводы}

В данной работе рассмотрен один из возможных подходов к разделению волновых полей на отраженную и дифрагированные компоненты. Определена модель выделения рассеянной компоненты поля, которая основывается на наличии в сейсмическом волновом поле доминирующей отраженной волны и в несколько раз меньшей по амплитуде волны дифрагированной. Так как соотношение энергий отраженной и дифрагированной волны может меняться то возможно тестирование работы программы и подстройка параметров для конкретных данных. Показан пример использования программы разделения на практическом материале.

\section{Библиография / References}

1. Landa E., Fomel S., Reshef M. 2008. Separation, imaging, and velocity analysis of seismic diffractions using migrated dip-angle gathers. SEG Las Vegas Annual Meeting, Expanded Abstract. p.2176-2180 
2. Ribet B., Yelin G., Serfaty Y., Chase D., Kelvin R., Koren Z. 2017. High resolution diffraction imaging for reliable interpretation of fracture systems. First Break. 35. p.43-47

3. Hoeber H, Pelissier M., Moser T.J, Klem Musatov K. 2017. Seismic diffractions: How it all began. First Break. 35. p.31-34

4. Koltanovsky L.E., Korolev A.E., Rossiyskaya E.M., Konstantin A. Smirnov K.A. 2017. Enhancing confidence in fracture prediction through advanced seismic data processing and analysis techniques. First Break. 35. p.49-53

5. Merzlikin D., Meckel T.A, Fomel1 S., Sripanich Y. 2017. Diffraction imaging of highresolution 3D P-cable data from the Gulf of Mexico using azimuthal plane-wave destruction First Break. 35. p.35-41

6. Pelissier M, Moser T.J, Yu C, Lang J, Sturzu I., Popovici A.M. 2017. Interpretation value of diffractions and sub-specular reflections - applications on the Zhao Dong field. First Break. 35. p.61-68

7. Zelewski G., Burnett W.A, Liu E, Johns M, Wu X, Zhang J, Skeith G. 2017. Diffraction imaging enhancement using spectral decomposition for faults, fracture zones, and collapse feature detection in a Middle East carbonate field. First Break. 35. p.55-60

8. Blias E. 2007. VSP wavefield separation: Wave-by-wave optimization approach. Geophysics, 72, p.T47-T55

9. Harlan W.S., Claerbout J.F., Rocca F., 1984. Signal/noise separation and velocity estimation. Geophysics, 49. p.1869-1880 